\title{
Multiview Sequential Learning and Dilated Residual Learning for a Fully Automatic Delineation of the Left Atrium and Pulmonary Veins from Late Gadolinium-Enhanced Cardiac MRI Images *
}

\author{
Guang Yang ${ }^{\dagger}$, Jun Chen, Zhifan Gao, Heye Zhang, Hao Ni, Elsa Angelini, Raad Mohiaddin, \\ Tom Wong, Jennifer Keegan ${ }^{*}$ and David Firmin ${ }^{*}$
}

\begin{abstract}
Accurate delineation of heart substructures is a prerequisite for abnormality detection, for making quantitative and functional measurements, and for computer-aided diagnosis and treatment planning. Late Gadolinium-Enhanced Cardiac MRI (LGE-CMRI) is an emerging imaging technology for myocardial infarction or scar detection based on the differences in the volume of residual gadolinium distribution between scar and healthy tissues. While LGE-CMRI is a well-established non-invasive tool for detecting myocardial scar tissues in the ventricles, its application to left atrium (LA) imaging is more challenging due to its very thin wall of the LA and poor quality images, which may be produced because of motion artefacts and low signal-to-noise ratio. As the LGE-CMRI scan is designed to highlight scar tissues by altering the gadolinium kinetics, the anatomy among different heart substructures has less distinguishable boundaries. An accurate, robust and reproducible method for LA segmentation is highly in demand because it can not only provide valuable information of the heart function but also be helpful for the further delineation of scar tissue and measuring the scar percentage. In this study, we proposed a novel deep learning framework working on LGE-CMRI images directly by combining sequential learning and dilated residual learning to delineate LA and pulmonary veins fully automatically. The achieved results showed accurate segmentation results compared to the state-of-the-art methods. The proposed framework leads to an automatic generation of a patient-specific model that can potentially enable an objective atrial scarring assessment for the atrial fibrillation patients.
\end{abstract}

\section{INTRODUCTION}

Noninvasive MRI is becoming an increasingly important tool for medical diagnosis and is widely used in many diverse clinical applications, e.g., grading and classifying brain tumours [1], [2] and evaluating volumes and function of the

* Research supported by British Heart Foundation Project Grant (Project Number: PG/16/78/32402).

$\dagger$ indicates corresponding author (e-mail: g.yang@imperial.ac.uk)

$\$$ indicates joint senior authors.

J. Chen, Z.F. Gao and H.Y. Zhang are with the Shenzhen Institutes of Advanced Technology, Chinese Academy of Sciences, Shenzhen, China.

$\mathrm{H}$. Ni is with the Department of Mathematics, University College London, WC1E 6BT, London, United Kingdom and the Alan Turing Institute, NW1 2DB, London, United Kingdom.

E. Angelini is with the Faculty of Medicine, Department of Surgery \& Cancer, Imperial College London, SW7 2AZ, London, United Kingdom.

T. Wong is with the Royal Brompton Hospital, SW3 6NP, London, United Kingdom.

G. Yang, R. Mohiaddin, J. Keegan and D. Firmin are with the National Heart and Lung Institute, Imperial College London, SW7 2AZ, London, United Kingdom and the Cardiovascular Research Centre, Royal Brompton Hospital, SW3 6NP, London, United Kingdom. heart ventricles [3]. Recently, Late Gadolinium-Enhanced Cardiac MRI (LGE-CMRI) has been established for visualizing and assessing myocardial infarction or fibrosis in the left ventricle [4]. This technique is based on the different wash-in and wash-out gadolinium contrast agent kinetics between healthy and scarred tissues. The hyper-enhanced regions in the LGE-CMRI images reflect the increased interstitial space of the myocardium with fibrosis, i.e., abnormal scar regions, while healthy ventricular myocardium is 'nulled' [5], [6]. LGE-CMRI has also been successfully acquired in patients with atrial fibrillation (AF) to detect native and post-ablation treatment scarring in the left atrium (LA) [7]. However, the LA wall is very thin and the diagnostic value of the LGE-CMRI scan is lowered. The nulling of signal from healthy tissue reduces the visibility of the LA wall boundaries and in addition, in the AF patient population, prolonged scanning time ( $\sim 10$ mins for whole-heart $3 \mathrm{D}$ LGE-CMRI), irregular breathing pattern and heart rate variability during the scan can result in poor image quality. High signal intensity from other structures, such as the aorta and mitral valve, can further complicate the detection and localization of the LA.

The delineation of the LA and the attached pulmonary veins $(\mathrm{PV})$ is of high clinical importance in patients with AF. Firstly, accurate LA segmentation can provide information on chamber size and function, and it is also essential for determining atrial scarring as a percentage of LA wall volume [8]. Secondly, PVs are a frequent source of the aberrant electrical activity causing AF and electrical isolation of the PV by scarring the surrounding tissue may eliminate the AF [9]. Precise delineation of the PV will therefore help to localize possible conduction gaps after ablation based treatment and may potentially be used to predict AF recurrence [10], [11]. Thirdly, the delineated LA and PV are potentially useful to guide the ablation procedure and ultimately to reduce the need for repeat procedures [12].

Most previous studies have used manual segmentation of the LA and PV. However, this is time-consuming, subjective and lacks reproducibility [12]-[16]. Knowles et al. [17] described a semi-automatic thresholding and region growing method for the delineation of the LA and PV, with the segmentation performed on first-pass Magnetic Resonance Angiography (MRA) images, in which LA, PV and other blood vessels were seen with high contrast. However, while the LA and PV segmentation from the MRA images may be relatively straightforward, the following registration between the MRA and LGE-CMRI images can limit the final accuracy of the delineation on the LGE-CMRI images. A statistical shape model is a widely used method to resolve the anatomy 
of the imaged organs in medical images, which is analogous to the active contour model, but also learns patterns of shape variability from a training set of manually annotated region of interests (ROIs) [18]. Karim et al. [19] proposed a statistical shape model based method to segment the LA and PV followed by manual corrections. The segmentation was performed on images acquired by a 3D balanced steady-state free precession (b-SSFP) acquisition, but this also requires subsequent registration between corresponding b-SSFP and LGE-CMRI images. Tao et al. [20] reported an automatic atlas based method that was coupled with level set based refinement on MRA images to delineate the LA and PV. More accurate delineation could be achieved via information gained from the MRA, but the method also suffered from the additional registration error. Yang et al. [21] utilized multi-atlas based whole heart segmentation to solve the problem and proposed a super-voxel based post-processing to achieve more accurate segmentation of the PV. Recently, deep learning based methods have attracted lots of interests mainly due to their performance and efficiency by leveraging the available big data and GPU computing. By using b-SSFP images, Mortazi et al. [22] developed a deep learning architecture, namely CardiacNET, to segment the LA and PV. This method could be potentially useful for segmenting the LA and PV from the LGE-CMRI images but to the best of our knowledge, this has not been done to date.

All of these previous studies have relied on either manual or partially manual procedures and/or an additional scanning sequence (for a better imaging of the heart anatomy) to segment the LA and PV. A fully automatic method directly working on the LGE-CMRI images is highly desirable to avoid tedious manual interference and possible registration error between anatomical cardiac scans and the LGE-CMRI. This would also reduce the scanning cost and the patient burden.

In this study, a novel deep learning based framework is designed to segment the LA and PV directly from the LGE-CMRI images, avoiding the need for an additional data acquisition for anatomical segmentation and subsequent registration. The proposed framework jointly learns the segmentation of the LA and PV and the correlation in-between axial slices of the 3D LGE-CMRI dataset via a sequential learning network. In addition, multiview information has been learned by two dilated residual learning networks from the sagittal and coronal views of the 3D LGE-CMRI dataset. Eventually, the learned representations of the sagittal and coronal views are integrated into the corresponding axial view to obtain the fused multiview features, which will be used to derive the final segmentation of the LA and PV. The following sections present the details of the proposed methodology, experimental setup, achieved results and followed by discussions and conclusion.

\section{METHODS}

\section{A. Patients and Data Acquisition}

Cardiac MR data were acquired in patients with longstanding persistent AF on a Siemens Magnetom Avanto 1.5T scanner (Siemens Medical Systems, Erlangen, Germany). Transverse navigator-gated 3D LGE-CMRI [7], [14], [23] was performed using an inversion prepared segmented gradient echo sequence (TE/TR $2.2 \mathrm{~ms} / 5.2 \mathrm{~ms}) 15$ minutes after gadolinium administration
(Gadovist_-gadobutrol, $0.1 \mathrm{mmol} / \mathrm{kg}$ body weight, Bayer-Schering, Berlin, Germany) [5]. The inversion time was set to null the signal from normal myocardium. Detailed scanning parameters are: $30-34$ slices at $(1.4-1.5) \times(1.4-$ $1.5) \times 4 \mathrm{~mm}^{3}$, reconstructed to $60-68$ slices at $(0.7-0.75) \times(0.7-$ $0.75) \times 2 \mathrm{~mm}^{3}$, field-of-view $380 \times 380 \mathrm{~mm}^{2}$. For each patient, prior to contrast agent administration, coronal navigator-gated 3D b-SSFP (TE/TR $1 \mathrm{~ms} / 2.3 \mathrm{~ms}$ ) data were acquired with the following parameters: $72-80$ slices at $(1.6-1.8) \times(1.6-$ $1.8) \times 3.2 \mathrm{~mm}^{3}$, reconstructed to $144-160$ slices at $(0.8-$ $0.9) \times(0.8-0.9) \times 1.6 \mathrm{~mm}^{3}$, field-of-view $380 \times 380 \mathrm{~mm}^{2}$. Both LGE-CMRI and b-SSFP data were acquired during free-breathing using a crossed-pairs navigator positioned over the dome of the right hemi-diaphragm with navigator acceptance window size of $5 \mathrm{~mm}$ and CLAWS respiratory motion control [24]. It is of note that for our deep learning framework, only LGE-CMRI data are required, and b-SSFP data are used only for comparison studies.

In order to test the efficacy of our deep learning framework and perform comparison studies, we retrospectively included 20 LGE-CMRI scans as an independent testing dataset (including randomly chosen 10 pre-ablation and 10 post-ablation scans) while the deep learning framework was trained on 80 randomly selected LGE-CMRI data. Ground truth of the LA and PV segmentation was manually delineated by an experienced physicist. All the patient recruitment and data acquisition were approved by the institutional review board in accordance with local ethics procedures.

\section{B. Sequential Learning}

In order to perform joint learning for the LA and PV segmentation from the axial view slice and the correlation in-between axial slices, we propose a sequential learning network (Figure 1) that consists of six convolutional layers for the encoder and decoder path respectively. In the encoder path, each convolutional layer is followed by a rectified linear unit (ReLU) layer and a local response normalization (LRN) layer to normalize the feature maps in order to improve the generalization performance of the network and speed up the training procedure. In addition, max-pooling layers are used to reduce the dimension of the feature maps. In the decoder path, three up-sampling layers are implemented via bilinear interpolation to recover the original image size, and the decoder is also incorporated convolutional and LRN layers. Each convolutional layer contains 12 kernels with size of $3 \times 3$ pixels. In addition, convolutional long-short term memory (ConvLSTM) [25] layers are embedded into the encoder-decoder network to account for inter-slices correlations. The ConvLSTM is a special recursive neural network architecture that can be defined mathematically as

$$
\begin{gathered}
i_{t}=\sigma\left(W_{x i} * x_{t}+W_{h i} * h_{t-1}+W_{c i} \circ c_{t-1}+b_{i}\right), \\
f_{t}=\sigma\left(W_{x f} * x_{t}+W_{h f} * h_{t-1}+W_{c f} \circ c_{t-1}+b_{f}\right), \\
c_{t}=f_{t} \circ c_{t-1}+i_{t} \circ \operatorname{ReLU}\left(W_{x c} * x_{t}+W_{h c} * h_{t-1}+b_{c}\right), \\
o_{t}=\sigma\left(W_{x o} * x_{t}+W_{h o} * h_{t-1}+W_{c o} \circ c_{t}+b_{o}\right) \\
h_{t}=o_{t} \circ \operatorname{ReLU}\left(c_{t}\right)
\end{gathered}
$$

where ' $*$ ' represents the convolutional operator and ' $\circ$ ' denotes the Hadamard product, $W$ terms denote weight matrices, $b$ terms denote bias vectors, $\sigma$ represents a sigmoid function and ReLU is used in our study instead of tanh. The ConvLSTM uses three gates including the input gate $i_{t}$, the 
forget gate $f_{t}$ and the output gate $o_{t}$, and memory cell $c_{t}$ represents an accumulator of the state information and $h_{t}$ denotes the hidden states. The input gate $i_{t}$ determines which values of current input $x_{t}$ should be saved to the current cell state $c_{t}$. The forget gate $f_{t}$ controls which values of previous cell state $c_{t-1}$ should be remained to the current cell state $c_{t}$. Which part of the latest cell output $c_{t}$ should be propagated to the final state $h_{t}$ is further controlled by the output gate $o_{t}$. In addition, 12 convolutional kernels are used with the size of $3 \times 3$ pixels. During the training procedure, the axial slices of the image are encoded via the encoder path that incorporates the ConvLSTM, which models the sequential dependencies between the axial slices. In addition, the output sequence of the ConvLSTM is up-sampled via the decoder path.

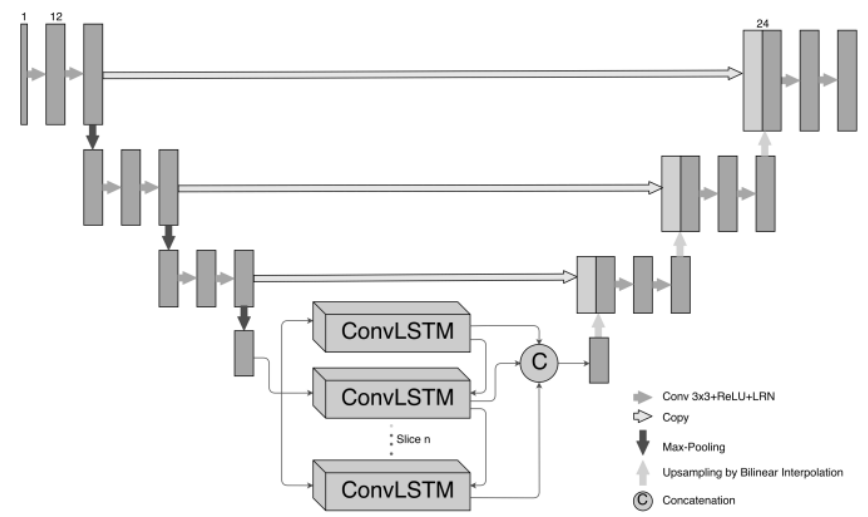

Figure 1: Schema of our sequential learning network.

\section{Dilated Residual Learning}

In order to learn the complementary information from the sagittal and coronal views, we propose to use a full convolutional neural networks (CNN) with shortcut connections that is similar to the residual network [26]. The full CNN consists of 4 convolutional layers, and each is followed by a ReLU layer and a LRN layer (Figure 2). In each convolutional layer, 12 convolutional kernels are used with the size of $3 \times 3$. In order to fully consider the complementary information from both sagittal and coronal views, we use the dilated convolution [27] instead of using the pooling layers that can avoid loss of useful information during the pooling operation. By using the dilated convolution, the receptive field is remained and the size of the feature maps is not reduced.

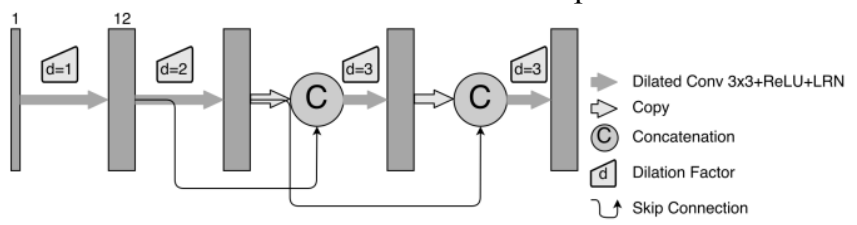

Figure 2: Schema of our dilated residual learning network.

Finally, the learned feature maps from the sagittal and coronal views need to be integrated with the feature maps obtained from the sequential learning to form the fused multiview features. In order to achieve this, two 3D volumes are created to store the learned feature maps from the sagittal and coronal view respectively. Then we slice them into multiple 2D axial slices, and concatenate them with the feature maps derived from the sequential learning of the axial view at their corresponding channels. Next, a convolutional operation is applied to these concatenated feature maps to get the fused multiview features. At the end, three convolutional layers are connected to perform the segmentation based on the fused multiview features. Two of them contain 24 kernels with the size of $3 \times 3$ and each is followed by a ReLU layer and a LRN layer. At the last layer, a $3 \times 3$ convolution is used to map each pixel to the desired segments, and the sigmoid activation function is used.

For the implementation, we used the Adam method [28] to perform the optimization with a mean squared error based loss function and decayed learning rate (initial learning rate was 0.001 and dropped to 0.000296 at the end). Our deep learning model was implemented using Tensorflow 1.2.1 on a Ubuntu 16.04 machine, and was trained and tested on an NVidia Tesla P100 GPU (3584 cores and 16GB).

\section{Comparison Study}

We compared our fully automatic deep learning framework with (1) a fully automatic multi-atlas based whole heart segmentation (WHS) method, and (2) a semi-automatic method coupled WHS and super-voxel based refinement (WHS+SV) [21], [29]. It is of note that in these two methods, both LGE-CMRI and b-SSFP data were used and required a registration operation to align the two sets of corresponding data, while for our proposed deep learning framework only LGE-CMRI images were required.

\section{E. Evaluation Metrics}

The output of our deep learning framework was binarized to a ROI mask for the LA and PV delineation. Same binary mask was created using the results obtained by the comparison methods. We used the evaluation metrics including pixel-wise accuracy (AC), sensitivity (SE), specificity (SP) and Dice score (DI). The evaluation metrics are defined using the number of true positives (TP), true negatives (TN), false positives (FP), and false negatives (FN) respectively as

$$
\begin{gathered}
\mathrm{AC}=(\mathrm{TP}+\mathrm{TN}) /(\mathrm{TP}+\mathrm{FP}+\mathrm{TN}+\mathrm{FN}), \\
\mathrm{SE}=\mathrm{TP} /(\mathrm{TP}+\mathrm{FN}), \\
\mathrm{SP}=\mathrm{TN} /(\mathrm{TN}+\mathrm{FP}), \\
\mathrm{DI}=2 \times \mathrm{TP} /(2 \times \mathrm{TP}+\mathrm{FN}+\mathrm{FP}) .
\end{gathered}
$$

Furthermore, statistical significances $(\mathrm{P}<0.05)$ were given by a two-sample Wilcoxon rank-sum test.

\section{RESULTS}

Figure 3 shows the convergence of the training and testing stages of our proposed framework, respectively.

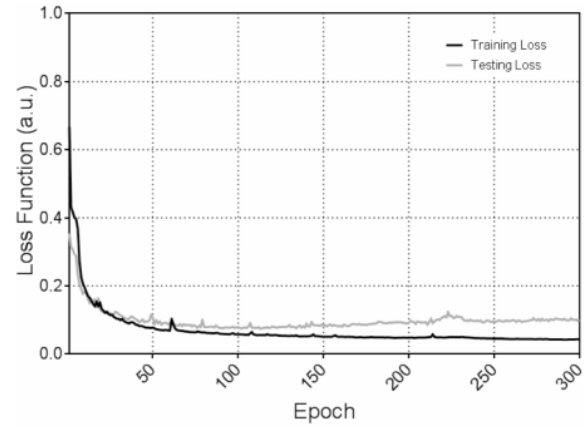

Figure 3: Training and testing loss functions. 
Figure 4 (d) displays the qualitative visualization of the LA and PV segmentation using our proposed framework, and compared to the manually drawn ground truth (Figure 4 (a)) and results obtained by the fully automatic WHS and semi-automatic WHS+SV methods (Figure 4 (b) and (c)). For this example case, compared to the ground truth, semi-automatic WHS-SV method segmented the left inferior PV (LIPV) most accurately (cyan arrows in Figure 4 (a-d)). For the right inferior PV (RIPV), both WHS-SV and our proposed framework performed better than the WHS method (green arrows in Figure 4 (a-d)). For the segmentation of the LA regions, our proposed framework provided a smoother and more accurate delineation compared to the ground truth (purple arrows in Figure 4 (a-d)).

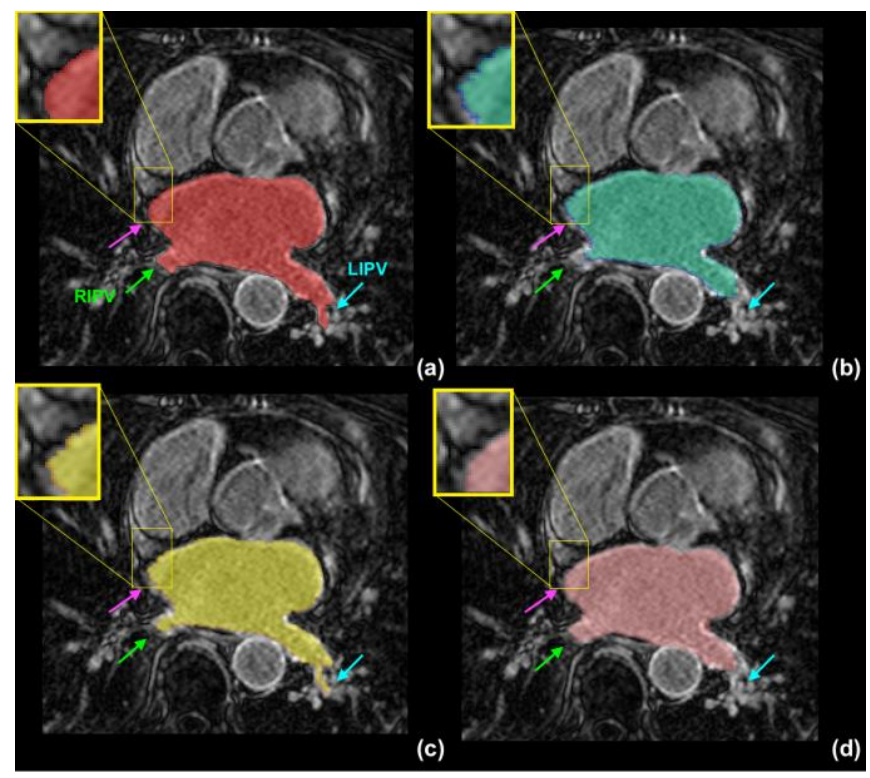

Figure 4: Qualitative visualization of the LA and PV segmentation.

Quantitative results including AC, SE, SP and DI are tabulated in Table 1. WHS $+\mathrm{SV}$ obtained slightly higher mean $\mathrm{AC}$ and DI, but there was no statistically significant difference found between the results obtained by the WHS+SV and our proposed framework (for the $\mathrm{DI}$ : $\mathrm{P}=0.072$ ). In addition, we found statistically significant differences between the WHS + SV and the WHS method (for the DI: $\mathrm{P}=2.04 \mathrm{E}-05$ ) and between our proposed framework and the WHS method (for the DI: $\mathrm{P}=0.011$ ).

Table 1: Quantitative evaluation results.

\begin{tabular}{lcccc}
\hline Methods & AC & SE & SP & DI \\
\hline \hline WHS & $0.998 \pm 0.001$ & $0.809 \pm 0.187$ & $0.999 \pm 0.001$ & $0.870 \pm 0.146$ \\
WHS+SV & $0.998 \pm 0.001$ & $0.853 \pm 0.099$ & $0.999 \pm 0.000$ & $0.908 \pm 0.067$ \\
Proposed & $0.997 \pm 0.002$ & $0.856 \pm 0.137$ & $0.999 \pm 0.001$ & $0.897 \pm 0.053$ \\
\hline
\end{tabular}

\section{DISCUSSIONS AND CONCLUSION}

In this work, we present a fully automatic deep learning framework to segment the LA and PV from LGE-CMRI images directly. By combining the sequential learning and dilated residual learning for extracting multiview features, our framework has shown favorable results for this challenging segmentation task. Validation of our framework has been performed against manually delineated LA and PV.
Compared to both a fully-automatic multi-atlas based WHS method and a semi-automatic WHS+SV method, our framework has demonstrated comparable accuracy when using only the LGE-CMRI data.

Compared to the WHS and the WHS+SV method, our deep learning based framework obtained smoother boundaries. This is due to the fact that the WHS may suffer from errors when registering the segmented anatomy to the LGE-CMRI data and these may produce such jagged artefacts in the delineated contour (Figure 4 (b) and (c)). Compared to the WHS-SV method, our proposed framework obtained comparable AC, SE and SP (Table 1). Although the DI obtained by our method is slightly worse than using the WHS+SV (Table 1), there was no statistically significant difference between the two. Moreover, although the WHS+SV method segmented the PV more accurately, the better delineations were only obtained at the distal regions of the PV (e.g., distal regions of the LIPV in Figure 4 (a) and (c)) that may not influence the final treatment planning or prognosis for the AF patients.

For the WHS+SV method, the connections between different axial slices were taken into account via the super-voxels over-segmentation, i.e., using the connectivity of super-voxels in 3D. Compared with WHS+SV with a manual interference, our deep learning based framework resolved such correlation in-between axial slices via the ConvLSTM, which is a robust and efficient recursive neural network. Such correlation will also be constrained via dilated residual learning for the sagittal and coronal views.

There are limitations of the current work. First, the performance of our proposed deep learning based framework did not rely on a comprehensive network parameters tuning; however, currently used network parameters defined by test and trials may cause possible overfitting of the trained models (Figure 3). This will be mitigated via further investigation on techniques that can reduce the overfitting, e.g., using early stopping [30]. Second, we may need to compare our proposed framework with other recently developed deep learning based segmentation methods, e.g., U-Net [31]. Although benchmarking of various deep learning methods for solving the LA and PV segmentation is beyond the scope of current study, it will be an interesting future study to compare with other deep learning based methods.

In conclusion, the proposed method makes it possible to create a patient-specific anatomical model for the LA and PV that is accredited by our efficient and objective segmentation. This will potentially enable an objective atrial scarring assessment for individual AF patients that is a promising future working direction.

\section{REFERENCES}

T. L. Jones et al., "Brain tumor classification using the diffusion tensor image segmentation (D-SEG) technique.," Neuro. Oncol., vol. 17, no. 3, pp. 466-476, Aug. 2014.

[2] G. Yang et al., "Discrimination between glioblastoma multiforme and solitary metastasis using morphological features derived from the p:q tensor decomposition of diffusion tensor imaging.," $N M R$ Biomed., vol. 27, no. 9, pp. 1103-1111, Sep. 2014.

[3] C. F. Mooij et al., "Reproducibility of MRI measurements of right 
ventricular size and function in patients with normal and dilated ventricles.," J. Magn. Reson. Imaging, vol. 28, no. 1, pp. 67-73, Jul. 2008.

[4] P. Kellman and A. E. Arai, "Cardiac imaging techniques for physicians: Late enhancement," J. Magn. Reson. Imaging, vol. 36, no. 3, pp. 529-542, Sep. 2012.

[5] J. Keegan et al., "Improved respiratory efficiency of 3D late gadolinium enhancement imaging using the continuously adaptive windowing strategy (CLAWS)," Magn. Reson. Med., vol. 71, no. 3, pp. 1064-1074, 2014.

[6] J. Keegan et al., "Dynamic inversion time for improved 3D late gadolinium enhancement imaging in patients with atrial fibrillation.," Magn. Reson. Med., vol. 73, no. 2, pp. 646-54, Feb. 2015.

[7] D. C. Peters et al., "Detection of pulmonary vein and left atrial scar after catheter ablation with three-dimensional navigator-gated delayed enhancement MR imaging: initial experience.," Radiology, vol. 243, no. 3, pp. 690-695, 2007.

[8] G. Yang et al., "Fully Automatic Segmentation and Objective Assessment of Atrial Scars for Longstanding Persistent Atrial Fibrillation Patients Using Late Gadolinium-Enhanced MRI," arXiv, May 2017.

[9] W. S. Lin et al., "Pulmonary vein morphology in patients with paroxysmal atrial fibrillation initiated by ectopic beats originating from the pulmonary veins: implications for catheter ablation.," Circulation, vol. 101, no. 11, pp. 1274-81, Mar. 2000.

[10] K. Higuchi et al., "Cardiac MRI assessment of atrial fibrosis in atrial fibrillation: implications for diagnosis and therapy.," Heart, vol. 100, no. 7, pp. 590-6, 2014.

[11] C. Sohns et al., "Quantitative magnetic resonance imaging analysis of the relationship between contact force and left atrial scar formation after catheter ablation of atrial fibrillation.," $J$. Cardiovasc. Electrophysiol., vol. 25, no. 2, pp. 138-45, Feb. 2014.

[12] R. Karim et al., "Evaluation of current algorithms for segmentation of scar tissue from late gadolinium enhancement cardiovascular magnetic resonance of the left atrium: an open-access grand challenge.," J. Cardiovasc. Magn. Reson., vol. 15, pp. 105-122, 2013.

[13] C. McGann et al., "Atrial fibrillation ablation outcome is predicted by left atrial remodeling on MRI," Circ. Arrhythmia Electrophysiol., vol. 7, no. 1, pp. 23-30, 2014.

[14] R. S. Oakes et al., "Detection and quantification of left atrial structural remodeling with delayed-enhancement magnetic resonance imaging in patients with atrial fibrillation," Circulation, vol. 119, no. 13, pp. 1758-1767, 2009.

[15] D. Perry et al., "Automatic classification of scar tissue in late gadolinium enhancement cardiac MRI for the assessment of left-atrial wall injury after radiofrequency ablation," in In SPIE Medical Imaging, 2012, p. 83151D.

[16] D. Ravanelli et al., "A novel skeleton based quantification and 3-D volumetric visualization of left atrium fibrosis using late gadolinium enhancement magnetic resonance imaging," IEEE Trans. Med. Imaging, vol. 33, no. 2, pp. 566-576, 2014.

[17] B. R. Knowles et al., "3-D visualization of acute RF ablation lesions using MRI for the simultaneous determination of the patterns of necrosis and edema," IEEE Trans. Biomed. Eng., vol. 57 , no. 6 , pp. $1467-1475,2010$

[18] T. F. Cootes et al., "Active Shape Models-Their Training and Application," Comput. Vis. Image Underst., vol. 61, no. 1, pp. 38 59, Jan. 1995.

[19] R. Karim et al., "A Method to Standardize Quantification of Left Atrial Scar from Delayed-Enhancement MR Images," Transl. Eng. Heal. Med. IEEE J., vol. 2, pp. 1-15, 2014.

[20] Q. Tao et al., "Fully automatic segmentation of left atrium and pulmonary veins in late gadolinium-enhanced MRI: Towards objective atrial scar assessment.," J. Magn. Reson. Imaging, vol. 44, no. 2, pp. 346-354, Jan. 2016

[21] G. Yang et al., "Multi-Atlas Propagation Based Left Atrium Segmentation Coupled with Super-Voxel Based Pulmonary Veins Delineation in Late Gadolinium-Enhanced Cardiac MRI," in SPIE Medical Imaging, 2017, pp. 1013313-1.

[22] A. Mortazi et al., "CardiacNET: Segmentation of Left Atrium and Proximal Pulmonary Veins from MRI Using Multi-view CNN," in International Conference on Medical Image Computing and
Computer-Assisted Intervention, 2017, pp. 377-385.

[23] D. C. Peters et al., "Recurrence of Atrial Fibrillation Correlates With the Extent of Post-Procedural Late Gadolinium Enhancement. A Pilot Study," JACC Cardiovasc. Imaging, vol. 2, no. 3, pp. 308-316, 2009.

[24] J. Keegan et al., "Navigator artifact reduction in three-dimensional late gadolinium enhancement imaging of the atria," Magn. Reson. Med., vol. 785, pp. 779-785, 2013.

[25] X. Shi et al., "Convolutional LSTM Network: A Machine Learning Approach for Precipitation Nowcasting," in NIPS, 2015.

[26] K. He et al., "Deep Residual Learning for Image Recognition," in 2016 IEEE Conference on Computer Vision and Pattern Recognition (CVPR), 2016, pp. 770-778.

[27] F. Yu and V. Koltun, "Multi-Scale Context Aggregation by Dilated Convolutions," in International Conference on Learning Representations, 2015.

[28] D. Kingma and J. Ba, "Adam: A Method for Stochastic Optimization," arXiv, Dec. 2014

[29] X. Zhuang and J. Shen, "Multi-scale patch and multi-modality atlases for whole heart segmentation of MRI," Med. Image Anal., vol. 31, pp. 77-87, Jul. 2016

[30] A. Géron, Hands-on machine learning with Scikit-Learn and TensorFlow: concepts, tools, and techniques to build intelligent systemsNo Title. O’Reilly, 2017.

[31] O. Ronneberger et al., "U-Net: Convolutional Networks for Biomedical Image Segmentation," in Medical Image Computing and Computer-Assisted Intervention, 2015, pp. 234-241. 